\title{
Real-World Clinical Experience with SGLT2 Inhibitors: Use of Special Screening Tool for Type 2 Diabetes Patients to Avoid Serious Adverse Events: A Single-Centre Prospective Study
}

\author{
Vishwa B. Unadkat ${ }^{a}$ Sandeep Sharma ${ }^{b}$ Ruchi Omar ${ }^{b}$ \\ aDepartment of Medicine, BAPS Shastriji Maharaj Hospital, Vadodara, India; ${ }^{b}$ Department of Epidemiology, \\ ICMR-National JALMA Institute of Leprosy and Other Mycobacterial Diseases, Agra, India
}

\section{Keywords \\ Effectiveness - Real world · Safety · Screening of patients · SGLT2 inhibitor · Type 2 diabetes}

\begin{abstract}
Background: Promising results of clinical trials involving SGLT2 inhibitors urge every clinician managing diabetes to use them. However, upcoming real-world data still show increased incidence of adverse events, but efficacy is comparable to clinical trials. Objectives: Genitourinary infection is the most commonly reported adverse effect with SGLT2 inhibitors. We evaluated effectiveness of patient screening protocol and advice of hygiene and hydration to avoid adverse effects of SGLT2 inhibitors in real-world setting. Method: This was a prospective observational longitudinal study which included consecutive subjects with uncontrolled T2DM recommended with SGLT2i after a simple screening protocol from December 2017 to November 2018. The adverse effects and metabolic parameters were evaluated at 1st, 3rd, 6th, and 12th months for each patient. Results: Of 413 patients recommended for SGLT2 inhibitors, 335 patients started the medication. At baseline, average age, glycosylated haemoglobin ( $\mathrm{HbA} 1 \mathrm{c})$, and weight were 53 years, 9.5\%, and $82 \mathrm{~kg}$,
\end{abstract}

respectively. Data of 332, 299, 270, and 231 patients were available at the $1 \mathrm{st}, 3 \mathrm{rd}, 6 \mathrm{th}$, and 12 th months for safety follow-up, respectively. Genitourinary tract infection was the most common adverse effect (8\%) followed by ketosis (4\%). Two patients needed to stop the drug permanently due to recurrent transient ischaemic attack and emphysematous pyelonephritis. Significant reduction in mean weight and HbA1c was observed at 6 months $(n=270): 2.9 \mathrm{~kg}$ and $1.1 \%$, respectively, and at 12 months $(n=231): 3.8 \mathrm{~kg}$ and $1.6 \%$, respectively. Conclusion: Simple screening protocol for patients considered for SGLT2i significantly reduced incidence of genitourinary adverse events. @ 2020 The Author(s)

Published by S. Karger AG, Basel

\section{Introduction}

Diabetes mellitus is enjoying an epidemic state around the world, mainly in countries like India. The global diabetes prevalence in 2019 is estimated to be $9.3 \%$ (463 million), rising to $10.2 \%$ (578 million) by 2030 , and $10.9 \%$ (700 million) by 2045 [1]. It is estimated that India is home to 77 million diabetics. Approximately $60 \%$ of patients diagnosed with diabetes have uncontrolled diabe- karger@karger.com www.karger.com/dde

Karger $\frac{1}{\%}$

GOPEN ACCESS
(C) 2020 The Author(s)

Published by S. Karger AG, Basel

This article is licensed under the Creative Commons AttributionNonCommercial-NoDerivatives 4.0 International License (CC BYNC-ND) (http://www.karger.com/Services/OpenAccessLicense). Usage and distribution for commercial purposes as well as any distribution of modified material requires written permission.
Vishwa B. Unadkat

Consultant Physician \& Diabetologist

BAPS Shastriji Maharaj Hospital

Vadodara 390012 (India)

vishw_u4u@yahoo.com 
tes. They require improvement in ongoing treatment to achieve good glycaemic control and prevent or delay the complications related to diabetes. SGLT2 inhibitors (SGLT2i) and GLP1RA improved diabetes treatment to a great extent. They provide good glycaemic control and weight reduction. Kidneys play a major role in the regulation of glucose reabsorption and maintenance of the overall metabolic balance in the body. Of $\sim 180 \mathrm{~g} /$ day of filtered glucose in healthy kidneys, $99 \%$ is reabsorbed [2, 3]. Glucose reabsorption in the kidney occurs through 2 types of transporters: the facilitative glucose transporters and an active sodium-glucose co-transporters (SGLTs) [4]. Among the different types of SGLTs, SGLT2 is expressed primarily in the kidneys, where it is found in the brush border membrane of the S1 segment of the proximal renal tubule and account for $90 \%$ of glucose reabsorption in kidneys [5]. SGLT2i decreases the renal threshold for glucose reabsorption leading to urinary glucose excretion and net caloric loss, which results in lowering of blood glucose level and weight loss. Use of SGLT2i

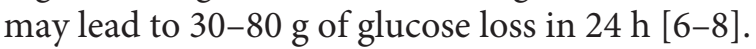

Dapagliflozin, canagliflozin, and Empagliflozin are SGLT2i, which are FDA and EMA approved and are available for clinical use in India. They are recommended for the treatment of type 2 diabetes as monotherapy or in combination with other antidiabetic drugs. There are many randomized and prospective trials which showed good glycaemic control and weight reduction benefits of these drugs $[9,10]$. Genitourinary infection is the most commonly reported adverse effect with SGLT2i in the clinical trials including these drugs. Diabetes patients are already at increased risk of genitourinary infections and use of SGLT2i might add significantly to this problem. Early real-world clinical data with SGLT2i suggest that effectiveness of SGLT2i was comparable with clinical trials $[11,12]$. However, adverse events were more in clinical practice than those observed in clinical trials. We designed this prospective observational study with the primary objective of evaluation of the efficacy of screening protocol and advice of hygiene and hydration to prevent adverse effects of SGLT2i and secondary objective of the efficacy of SGLT2i in glycaemic control and weight loss in patients with type 2 diabetes in real-world clinical practice.

\section{Materials and Methods}

This observational prospective longitudinal study was conducted in outpatient clinic of medicine department of a secondary care hospital in Vadodara (Gujarat), India. Consecutive patients with uncontrolled type 2 diabetes $(\mathrm{HbAlc}>8.5)$ were subjected to clinical screening protocol before starting SGLT2i attended by a single consultant from December 1, 2017, to November 30, 2018. Total 413 patients were considered for SGLT2i. After screening, 338 patients were recommended to start SGLT2i regimen, and actually 335 patients initiated SGLT2i therapy. Clinical screening protocol included the parameters mentioned below: (a) patients who were able to/willing to drink ample amount of water, (b) had peripheral pulses palpable with good volume, (c) willing to/able to keep personal hygiene in private parts, and (d) no history of recurrent UTI/obstructive uropathy. Patients with fatty liver and eGFR up to $45 \mathrm{~mL} / \mathrm{min}$ were also included. Patients with or without complication related to diabetes, who had newly detected diabetes or on oral medications, and those who were not on insulin were included. Pregnant and lactating women were also excluded. Every patient visited a diabetes educator after getting final prescription and reinforced about maintaining hydration and good personal hygiene. Home-based sugar monitoring, ketosis symptoms, and urine ketone screening were explained to each patient. Follow-up data were collected at 1, 3, 6, and 12 months. Fasting blood sugar, postprandial blood sugar, and weight were obtained at each follow-up visit. $\mathrm{HbA1C}$ was measured at 3,6 , and 12 months. HbA1c was analysed by high-performance liquid chromatography method using Bio-Rad D10 analyser. eGFR/UACR was measured at the baseline, 6, and 12 months.

Adverse effects were monitored and urine laboratory test that is microscopy and culture were performed of symptomatic patients. Female patients were evaluated by gynaecological examination. HbA1c was measured at $0,3,6$, and 12 months. Adverse effects were considered severe when clinical decision was taken to stop SGLT2i permanently, mild to moderate when SGLT2i were continued or stopped temporarily. Patients were prescribed with dapagliflozin, empagliflozin, or canagliflozin. We analysed the efficacy and adverse effect profile as a class effect rather than individual drug effect. Informed written consent was obtained from all the patients. An independent ethics committee reviewed and approved the study protocol and informed consent form before screening.

\section{Results}

\section{Patient Disposition}

Totally 413 patients were considered for SGLT2i. After screening, 338 patients were recommended to start SGLT2i regimen, 335 patients initiated SGLT2i regimen, and remaining 3 denied SGLT2i regimen due to cost. Among 75 patients who failed screening, 10 patients had weak pulses, 47 patients had history of recurrent UTI/obstructive uropathy, and 18 patients were not able to/willing to maintain personal hygiene/hydration with their current lifestyle. Of 335 patients, 332, 299, 270 , and 231 patients came to OPD for follow-up at 1,3, 6 , and 12 months, respectively. Patients were evaluated for infection/ketosis at each follow-up visit. Urine examination was performed for the patients who had the symptoms. 
Table 1. Baseline characteristics $(n=413)$

\begin{tabular}{lc}
\hline Characteristics & Mean \\
\hline Age, years & 53 \\
Weight, kg & 82 \\
HbA1c, \% & 9.5 \\
BMI, kg/m ${ }^{2}$ & 27.3 \\
Duration of diabetes, years & 7 \\
Male/female & 1.05 \\
UACR $>30$ mg/g Cr, $n$ & 101 \\
eGFR $<60$ but $>45$ mL min, $n$ & 36 \\
Patients on various drugs, $n(\%)$ & \\
$\quad$ Metformin & $330(98.50)$ \\
Sulfonylureas & $216(64.47)$ \\
Pioglitazone & $48(14.32)$ \\
DPP4i & $138(41.19)$ \\
AGIs & $12(3.58)$ \\
GLP1RA & $10(2.98)$ \\
No data/new patient & $18(5.37)$ \\
\hline
\end{tabular}

DPP4i, dipeptidyl peptidase-4; AGIs, alpha glucosidase inhibitors; GLP-1RA, glucagon-like peptide 1.

\section{Patients' Demographic Characteristics}

The average age, weight, and BMI of the patients were 53 years, $82 \mathrm{~kg}$, and $27.3 \mathrm{~kg} / \mathrm{m}^{2}$, respectively. Baseline characteristics are shown in Table 1. At baseline, average HbA1c was 9.5\%. Average duration of diabetes among patients was 7 years. UACR of $>30 \mathrm{mg} / \mathrm{g}$ Cr was observed in 101 patients and eGFR of $<60$, but $>45 \mathrm{~mL}$ min was observed in 36 patients at baseline. Most of the patients were on metformin (98.5\%), sulfonylureas (64\%), DPP4i (41\%), and pioglitazone (14\%) at baseline along with others: AGIs (4\%) and GLP1RA (3\%). A few patients (5\%) were either new or did not have any record for the treatment. Baseline antidiabetic drugs used in the treatment of these patients are given in Table 2.

\section{Efficacy End Points}

In this observational study, real-world data regarding efficacy and safety of SGLT2i in diabetic patients were collected. At each follow-up, fasting blood sugar and postprandial blood sugar were reduced from the baseline. Average $\mathrm{HbA} 1 \mathrm{c}$ reduction at the end of 6 and 12 months from baseline was 1.1 and 1.6, respectively. Weight loss at the end of 6 and 12 months from baseline was 2.9 and 3.8 $\mathrm{kg}$, respectively (Table 3 ).

\section{Safety End Points}

Only 2 severe adverse effects (SAEs) were observed during this study. One patient, female of 62 years, devel-
Table 2. Patients taking antidiabetic drugs

\begin{tabular}{lc}
\hline Treatment & Patients, $n$ \\
\hline Patients on single drugs & \\
Metformin & 12 \\
SUs & 5 \\
Patients on combination of drugs & 52 \\
Metformin + DPP4i & 116 \\
Metformin + SUs & 6 \\
Metformin + GLP-1a & 24 \\
Metformin + pioglitazone & 7 \\
Metformin + AGIs & 65 \\
Metformin + SUs + DPP4i & 5 \\
Metformin + SUs + AGIs & 4 \\
Metformin + SUs + GLP-1a & 21 \\
Metformin + SUs + DPP4i + pioglitazone & 18 \\
No data available & 335 \\
Total & \\
\hline
\end{tabular}

SU, sulfonylurea; DPP4i, dipeptidyl peptidase-4; AGIs, alpha glucosidase inhibitors; GLP-1a, glucagon-like peptide 1 agonist.

oped recurrent right facial and arm weakness ( 3 times in 10 days). She had severe atherosclerotic stenosis of left carotid artery and underwent successful endarterectomy. The other patient was female of 59 years, diabetic for 6 years without prior history of UTI and her HbA1c, was 8.3, developed pyelonephritis after 2 months of SGLT2I initiation and needed urosurgical intervention. As evident from Table 4, we have discussed some parameters with the follow-ups. However, the details of other adverse events are listed in Table 5.

In all patients who reported 1 or other side effects, SGLT2i was stopped temporarily, except in patients who developed pyelonephritis and TIA, in whom the medicine was stopped permanently. Rest all patient could restart the drug within 2 to 4 weeks of time.

\section{Discussion}

Good glycaemic control is an important step in diabetes management to prevent diabetes-associated complications. Of late, major guidelines recommend SGLT2i inhibitors and GLP1R agonists after lifestyle changes and metformin, even considered as 1st line therapy for obese diabetic patients with ASCVD and DKD as landmark trials, showed their effectiveness in such patients. SGLT2i are preferred since they are orally active and due to its unique beta cell sparing action. However, they also in-
40

Dubai Diabetes Endocrinol J 2020;26:38-43 DOI: $10.1159 / 000510262$
Unadkat/Sharma/Omar 
Table 3. Efficacy end points (reduction from baseline)

\begin{tabular}{lllll}
\hline Parameter & $\begin{array}{l}\text { 1st follow-up } \\
\text { (1 month) }\end{array}$ & $\begin{array}{l}\text { 2nd follow-up } \\
\text { (3 months) }\end{array}$ & $\begin{array}{l}\text { 3rd follow-up } \\
\text { (6 months) }\end{array}$ & $\begin{array}{l}\text { 4th follow-up } \\
\text { (12 months })\end{array}$ \\
\hline Reduction in FBS, mg/dL & 23 & 42 & 53 & 72 \\
Reduction in PPBS, mg/dL & 38 & 70 & 82 & 90 \\
Reduction in weight, kg & 0.9 & 2.1 & 2.9 & 3.8 \\
Reduction in HbA1c & & 0.84 & 1.1 & 1.6 \\
\hline
\end{tabular}

FBS, fasting blood sugar; PPBS, postprandial blood sugar.

Table 4. Safety end points

\begin{tabular}{lllll}
\hline Parameter & $\begin{array}{l}\text { 1st follow-up } \\
(1 \text { month })\end{array}$ & $\begin{array}{l}\text { 2nd follow-up } \\
\text { (3 months) }\end{array}$ & $\begin{array}{l}\text { 3rd follow-up } \\
\text { (6 months) }\end{array}$ & $\begin{array}{l}\text { 4th follow-up } \\
(12 \text { months })\end{array}$ \\
\hline Genitourinary problem & 7 & 17 & 3 & 0 \\
Weakness & 2 & 2 & 0 & 3 \\
GI upset & 1 & 1 & 0 & 1 \\
Allergy & 0 & 0 & 0 & 0 \\
Ketosis & 5 & 7 & 1 & 0 \\
\hline
\end{tabular}

Table 5. Details of patients who developed side effects

\begin{tabular}{|c|c|c|c|c|c|}
\hline \multirow[t]{2}{*}{ Type of side effect $(N)$} & \multirow{2}{*}{$\begin{array}{l}\text { Gender } \\
\text { male } N(\%) \\
\text { female } N(\%)\end{array}$} & \multicolumn{2}{|c|}{ Age distribution, years } & \multicolumn{2}{|c|}{ Duration of diabetes } \\
\hline & & $\begin{array}{l}45-60 \\
N(\%)\end{array}$ & $\begin{array}{l}60-75 \\
N(\%)\end{array}$ & $\begin{array}{l}<5 \text { years } \\
N(\%)\end{array}$ & $\begin{array}{l}>5 \text { years } \\
N(\%)\end{array}$ \\
\hline Genitourinary (27) & $\begin{array}{l}\text { M } 3(11.11) \\
\text { F } 24(88.89)\end{array}$ & $\begin{array}{c}1(33.33) \\
24(100)\end{array}$ & $\begin{array}{l}2(66.67) \\
0(0)\end{array}$ & $7(25.93)$ & $20(74.07)$ \\
\hline Gastrointestinal (3) & $\begin{array}{l}\text { M } 2(66.67) \\
\text { F } 1(33.33)\end{array}$ & $\begin{array}{l}0(0) \\
0(0)\end{array}$ & $\begin{array}{l}2(100) \\
1(100)\end{array}$ & $0(0)$ & $3(100)$ \\
\hline Ketosis (13) & $\begin{array}{l}\text { M } 5(38.46) \\
\text { F } 8(61.54)\end{array}$ & $\begin{array}{l}4(80.00) \\
4(50)\end{array}$ & $\begin{array}{l}1(20) \\
4(50)\end{array}$ & $9(69.23)$ & $4(30.77)$ \\
\hline Weakness (7) & $\begin{array}{l}\text { M } 4(57.14) \\
\text { F } 3(42.86)\end{array}$ & $\begin{array}{l}2(50) \\
1(33.33)\end{array}$ & $\begin{array}{l}2(50) \\
2(66.67)\end{array}$ & $6(85.71)$ & $1(14.29)$ \\
\hline
\end{tabular}

crease glycosuria. It is a double-edged sword: it reduces weight due to calorie loss and predisposes a patient to the genitourinary infection.

This study was aimed at appropriate selection of patients for which SGLT2i would provide beneficial effects without undue increase in infection. Of 413 eligible subjects, $18 \%$ subjects failed simple clinical screening and were not recommended SGLT2i. To our knowledge, this is the 1st report applying a simple screening protocol for diabetes patients before recommendation of SGLT2i.
During follow-up of 1 year, $13.7 \%$ female subjects and $3 \%$ male subjects developed genitourinary infection. Other real-world studies reported higher GU infection rates including Gill et al. [13] (female 29.1\% and males 17.5\%) and Leiter et al. [14] (female 13.9\%, male 9.5\%) than our study. However, Mathieu et al. [15] reported cumulative incidence of GU infection $<6 \%$. Similar to our findings, incidence of GU infections was more in females in abovementioned studies. 
Other common side effects seen in the study were ketosis (4\% [13 patients]) and weakness (2\% [7 patients]). Two patients having weakness stopped medicine. No allergic reactions were noted during the study. Simple screening protocol for patients before recommending SGLT2i led to decrease in the adverse events. However, 2 patients developed serious adverse events, including TIA and emphysematous pyelonephritis and needed intervention. Post hoc analysis showed that 28\% (94/335) of the patients had history of established ASCVD (CAD/ PAD/CVD). The patient who developed TIA was already on ASA and statin. Of note, only 2 patients, developed emphysematous pyelonephritis and TIA, were required to stop medicine permanently. Other subjects who stopped medication due to $\mathrm{AE}$ re-started it when the $\mathrm{AE}$ were managed. We observed that SGLT2i reduced the $\mathrm{HbA1c}(1.6 \%)$ and led to weight reduction $(3.9 \mathrm{~kg})$ in patients with type 2 diabetes, which was comparable to other clinical trials and real-world studies.

Treating physician may consider carotid and urinary tract imaging in selected ASCVD patients who would be considered for SGLT2i. However, imaging is expansive and may not be possible for resource-deficient patient population. Future real-world clinical experience may unearth additional factors leading to better prescribing information on this useful but risky class of drug.

\section{Conclusion}

This is an observational prospective study which shows that the application of simple screening protocol among patients considered for SGLT2 inhibitor reduces the incidence of adverse events as compared to other studies.
With the gradual increase in our experience of using these groups of drugs, many adverse events seem to be preventable using this screening protocol. We can further evaluate this screening protocol by using more robust study design. Let us hope that our experience in the upcoming years will guide us better to see any extra precaution needed before starting these extremely useful, but potentially risky class of drug.

\section{Acknowledgements}

The authors would like to thank the patients for their participation and staff of the hospital for help and support.

\section{Statement of Ethics}

Informed written consent was obtained from all the patients. An independent ethics committee reviewed and approved the study protocol and informed consent form before screening.

\section{Conflict of Interest Statement}

The authors declare that they have no conflicts of interest.

\section{Funding Sources}

We certify that this article has not been funded by any funding agency.

\section{Author Contributions}

All authors contributed to this study.

\section{References}

1 Saeedi P, Petersohn I, Salpea P, Malanda B, Karuranga S, Unwin N, et al. Global and regional diabetes prevalence estimates for 2019 and projections for 2030 and 2045: results from the International Diabetes Federation diabetes atlas, 9. Diabetes Res Clin Pract. 2019 Nov; $157: 107843$.

2 Wright EM. Renal Na+-glucose cotransporters. Am J Physiol Renal Physiol. 2001 Jan; 280(1):F10-18.

3 Bakris GL, Fonseca VA, Sharma K, Wright EM. Renal sodium-glucose transport: role in diabetes mellitus and potential clinical implications. Kidney Int. 2009 Jun;75(12):1272-7.
4 Navale AM, Paranjape AN. Glucose transporters: physiological and pathological roles. Biophys Rev. 2016 Mar;8(1):5-9.

5 Bakris GL, Fonseca VA, Sharma K, Wright EM. Renal sodium-glucose transport: role in diabetes mellitus and potential clinical implications. Kidney Int. 2009 Jun;75(12):1272-7.

6 Komoroski B, Vachharajani N, Boulton D, Kornhauser D, Geraldes M, Li L, et al. Dapagliflozin, a novel SGLT2 inhibitor, induces dose-dependent glucosuria in healthy subjects. Clin Pharmacol Ther. 2009 May;85(5): 520-6.
7 Liu JJ, Lee T, DeFronzo RA. Why Do SGLT2 inhibitors inhibit only $30-50 \%$ of renal glucose reabsorption in humans? Diabetes. 2012 Sep;61(9):2199-204.

8 Ribola FA, Cançado FB, Schoueri JH, De Toni VF, Medeiros VH, Feder D. Effects of SGLT2 inhibitors on weight loss in patients with type 2 diabetes mellitus. Eur Rev Med Pharmacol Sci. 2017 Jan;21(1):199-211.

9 Bailey CJ, Gross JL, Pieters A, Bastien A, List JF. Effect of dapagliflozin in patients with type 2 diabetes who have inadequate glycaemic control with metformin: a randomised, double-blind, placebo-controlled trial. Lancet. 2010 Jun 26;375(9733):2223-33. 
10 Søfteland E, Meier JJ, Vangen B, Toorawa R, Maldonado-Lutomirsky M, Broedl UC. Empagliflozin as add-on therapy in patients with type 2 diabetes inadequately controlled with linagliptin and metformin: a 24-week randomized, double-blind, parallel-group trial. Diabetes Care. 2017 Feb;40(2):201-9.

11 Tamez-Perez HE, Delgadillo-Esteban E, SoniDuque D, Hernández-Coria MI, Tamez-Peña AL. SGLT2 inhibitors as add on therapy in type 2 diabetes: a real world study. J Diabetes Metab Disord. 2017 Jun;16:27.
12 McGovern A, Feher M, Munro N, de Lusignan S. Sodium-glucose co-transporter 2 (SGLT2) inhibitor: comparing trial data and real-world use. Diabetes Ther. 2017 Apr;8(2): 365-76.

13 Gill HK, Kaur P, Mahendru S, Mithal A. Adverse effect profile and effectiveness of sodium glucose co-transporter 2 inhibitors (SGLT2i): a prospective real-world setting study. Indian JEndocr Metab. 2019;23:50-5.
14 Leiter LA, Langslet G, Vijapurkar U, Davies MJ, Canovatchel W. Simultaneous reduction in both $\mathrm{HbAlc}$ and body weight with canagliflozin versus glimepiride in patients with type 2 diabetes on metformin. Diabetes Ther. 2016 Jun;7(2):269-78.

15 Mathieu C, Ranetti AE, Li D, Ekholm E, Cook W, Hirshberg B, et al. Randomized, doubleblind, phase 3 trial of triple therapy with dapagliflozin add-on to saxagliptin plus metformin in type 2 diabetes. Diabetes Care. 2015 Nov;38(11):2009-17. 